\title{
How an understudied trait has skewed autism studies for decades
}

\author{
BY LAURA DATTARO
}

27 OCTOBER 2021

\section{Listen to this story:}

https://www.spectrumnews.org/wpcontent/uploads/2021/10/audio-87690b15-bc18-4668-9d4a-9f9fa0a8fedb-encodings.mp3

About 15 years ago, psychologist Geoff Bird began to question a long-held tenet in autism research: the idea that all autistic people struggle with empathy.

For Bird, it just didn't make sense. In his experience, both autistic and non-autistic people vary widely in their ability to sense or feel another person's emotions - the scientific definition of empathy. And yet there was a body of research suggesting that autistic people are frequently unable to intuit what another person feels.

Two of Bird's colleagues proposed a solution to the riddle: What if, instead of lacking empathy, some autistic people can't recognize their own emotions, a little-known trait called alexithymia? Wouldn't that impair their ability to share someone else's?

Bird was skeptical but joined them on a research project - the results of which, published in 2010, shocked him: Among both autistic and non-autistic men, those with weaker brain responses to images of another person in pain had higher levels of alexithymia. After adjusting for alexithymia which was not routinely done - the empathetic brain responses in the two groups showed no differences at all.

"I instantly thought, 'Wow,' and realized that this alexithymia hypothesis had the potential to be incredibly significant in autism," says Bird, professor of cognitive neuroscience at the University of Oxford in the United Kingdom.

The 'alexithymia hypothesis,' if correct, may not only explain the broad range of emotion- 


\section{Spectrum | Autism Research News}

https://www.spectrumnews.org

processing difficulties autistic people appear to have - including, sometimes, none. It could also shake up how autism is screened for, diagnosed, treated and even defined.

Listen to a Spectrum webinar with Zachary Williams about measuring alexithymia.

"It's quite a novel concept," says Stephen Edwards, senior lecturer in psychology at Federation University Australia in Ballarat, who is developing a therapy to lessen alexithymia traits. And it comes with an important corollary: Any research into emotion processing in autistic people needs to measure and control for alexithymia, as is done with intelligence quotients, Bird and others say. Otherwise, alexithymia - which occurs in about half of autistic people, compared with 5 percent of neurotypical people - could be a major confounding factor in many autism studies.

Despite alexithymia's prevalence among autistic people, it may be unfamiliar to many autism researchers, Bird says. But a slew of new tools are poised to help them measure the trait more routinely - potentially reshaping emotion research in the process. Teams that have already heeded the call are discovering that accounting for alexithymia makes some apparent emotion-processing differences between autistic and non-autistic people disappear.

\section{Testing a hypothesis:}

Two psychiatrists coined the term 'alexithymia' in 1972, creating a mashup of Greek words meaning 'lack of words for emotions.' Today, it's defined by three characteristics: difficulty identifying and describing your own feelings and, although most research focuses on the former, a thinking pattern that emphasizes the external world over an internal one. Alexithymia can affect all elements of emotion-processing, including recognizing facial expressions and other people's emotions, and even emotional responses to music.

A few years after those first surprising results, Bird and a colleague articulated the hypothesis that alexithymia might explain some of the emotion-processing difficulties associated with autism and their variation among autistic people.

"It was the move from just saying, 'Shrug, all autistic people are different,' to say, 'OK, what's explaining the variance?"' Bird says.

Since then, researchers have found that alexithymia - more so than autism traits - can predict a person's eye-gaze patterns, the intensity of her response to or ability to recognize facial expressions, and even her tendency to share resources, a marker of prosocial behavior.

Bird is married to Jennifer Cook, senior fellow at the University of Birmingham in the U.K.. The two keep separate research lives, they say, but when Cook set up her own lab three years ago, measuring alexithymia became a founding principle. "Accounting for alexithymia is one of those 


\section{Spectrum | Autism Research News}

https://www.spectrumnews.org

things we just do in our studies now," she says.

Cook's work has lent support for Bird's theory. In a study published earlier this year, she and her colleagues asked 60 alexithymia-matched autistic and non-autistic adults to look at a series of dots animated to look like moving faces and rate them as angry, happy or sad. The two groups were equally adept at recognizing most emotions, the team found. Of note, autistic people struggled more than their non-autistic peers with recognizing anger, suggesting that something about that emotion is different for people with autism.

Illustration by Alexander Glandien

"Alexithymia is really responsible for lots of the difficulties autistic individuals have with emotion recognition," says Connor Keating, a graduate student in Cook's lab at the University of Birmingham in the U.K., who worked on the animation study and is dedicating his doctoral work to testing the alexithymia hypothesis. "And maybe that can explain loads of the difficulties that we have in the literature before we knew about alexithymia."

\section{Measuring up:}

Keating and others typically measure alexithymia with a self-report questionnaire called the 20-item Toronto Alexithymia Scale (TAS-20), which was created in 1994. Among non-autistic people, the higher the score, the more difficulty someone has recognizing their own emotions.

Among autistic people, though, it's not so straightforward. Researchers validated the tool in a small group of autistic people in 2005, but an independent team failed to replicate those results earlier this year. That team found that a condensed, eight-question version of the scale, which they dubbed the General Alexithymia Factor Score (GAFS-8), performed better than the TAS-20 in autistic adults.

Two newer scales, the Toronto Structured Interview for Alexithymia and the Perth Alexithymia Questionnaire (PAQ), are potentially even more accurate, says Zachary Williams, a medical and doctoral student at Vanderbilt University in Nashville, Tennessee, who helped create the GAFS-8.

A 2020 comparison of several alexithymia measures bears this out: Results on the TAS-20 may, at least in part, reflect a person's current levels of distress, the study showed, and not her baseline emotion processing - a problem not seen with the PAQ or another screen, the Bermond-Vorst Alexithymia Questionnaire.

Regardless, researchers need to parse how different measures affect their results, says Williams, 


\section{Spectrum | Autism Research News}

https://www.spectrumnews.org

who is autistic himself. "Whether alexithymia appears to predict a group difference more than autistic traits depends greatly on which measures are used to quantify autistic traits, alexithymia and the outcome of interest."

All of these measures rely exclusively on self-reports - a method that can be unreliable. So some researchers are working on creating more objective tools based on physiological responses. For instance, people with bigger surges in electrical activity across the skin - known as skin conductance - after viewing emotional images also score more highly on the TAS-20, even after controlling for autism traits, Cook and Keating demonstrated earlier this year.

The results jibe with theories that people with alexithymia have trouble distinguishing which situations require strong emotional responses, and so are frequently in a state of high stress. But they also run counter to the popular idea that alexithymic people are not in tune with their physical responses to emotions: According to their skin conductance responses, people with high alexithymia were just as good at rating their emotional responses as people with lower levels.

Bird, who is developing a measure based on skin conductance, heart rate and pupil dilation, has found that people who self-report alexithymia traits show a greater distinction between their own ratings of their emotional responses to images and pupil dilation.

Ideally, researchers will be able to combine questionnaires and other measures into a standardized set of tests for alexithymia, Keating says. "There's still a long way to go in terms of measuring alexithymia. We all need to come together and find some better ways to measure it."

\section{Improving health:}

Even with better tools to measure alexithymia, researchers face a challenge: recruiting autistic and non-autistic people who are matched on that trait. Still, the effort is worth it, proponents of the alexithymia hypothesis say. Not accurately measuring and accounting for alexithymia may be clouding our understanding of autism itself, which could affect who gets referred for a diagnosis and, coming full circle, who is included in research studies.

Because alexithymia seems to drive some traits in autism, some professionals who refer children for evaluations, such as teachers or pediatricians, may miss autistic children who lack emotionprocessing difficulties, says Andrew Surtees, lecturer in psychology at the University of Birmingham in the U.K.

And although alexithymia isn't measured as part of the autism diagnostic process, high alexithymia traits alone can push someone over the clinical cutoff on the gold-standard Autism Diagnostic Observation Scale (ADOS), Bird has shown. As a result, studies that use only the ADOS to identify autistic participants may inadvertently include non-autistic people with alexithymia in their cohort. 


\section{Spectrum | Autism Research News}

https://www.spectrumnews.org

Distinguishing between the two conditions can be difficult for diagnosticians to work out, Surtees says, but adding an explicit alexithymia screen to the process could help — which would benefit not only research projects but those individuals as well.

"For the people who are working with that autistic person trying to make their life better, or help them make their life better," Surtees says, "that information can be really crucial."

Identifying and targeting alexithymia in autistic people could also improve their mental health, because the trait is associated with increased rates of social-communication difficulties, anxiety and depression. And it could help clinicians identify who would benefit from which supports, particularly because some forms of therapy, such as cognitive behavioral therapy, may be less effective in people with high levels of alexithymia, Surtees says. Edwards is launching a study to assess whether a mimicry task can increase emotion-recognition ability and thereby ease distress in people with alexithymia and autism.

"If we can say this autistic person has alexithymia and this one hasn't, and we know this person with alexithymia will have his whole constellation of problems that the person without alexithymia won't," Bird says, "we're already better able to help those two individuals."

Cite this article: https://doi.org/10.53053/GNRQ3144 